\title{
Low Proficiency Does Not Mean Ab Initio: A Methodological Footnote for Linguistic
}

\section{Transfer Studies}

\section{Abstract:}

The goal of this brief article is to highlight a specific methodological consideration pertaining to the examination of linguistic transfer in sequential language acquisition: when and how can transfer be meaningfully disentangled from issues pertaining to developmental trajectories of the target language? While this methodological issue is relevant for all transfer studies irrespective of learner type or linguistic domain of inquiry, herein, we focus on a set of third language acquisition data. We examine the domain of negative quantifiers 'nobody/nothing' and negative polarity items 'anybody/anything' by Catalan-Spanish early bilinguals learning English as the L3 in adulthood. We offer two group analyses. The first is the superset of low beginner proficiency speakers (all participants taking part in a specially designed English course) and then a subset group (only those who were true ab initio L3 learners; that is, with no previous study of English). The analyses combine to show that exposure matters beyond proficiency; that is, even when proficiency is held constant at very low levels, low proficiency L3 learners who have had some instruction/exposure to an L3 pattern differently from truly $a b$ initio L3-learners. We discuss how this reality complicates isolating L3-transfer proper from effects of L3-development/acquisition and thus, by extension, to all cases of transfer such as adult and child L2.

Key words: L3/Ln acquisition, Morphosyntactic transfer, Exposure, Proficiency 


\section{Introduction}

One of the most enduring questions in non-native language acquisition concerns the role that previous language experience plays in subsequent acquisition. The majority of the relevant work has studied this by examining L1 transfer/cross-linguistic influence in sequential second language (L2) acquisition in adulthood. Although the construct of transfer proliferates in virtually all theoretical paradigms, studying it has taken center stage in formal (generative) linguistic approaches to L2 acquisition since its inception in the early 1980s (e.g., White, 1989, 2003 for review). Although the terms cross-linguistic influence (CLI) and transfer are often used interchangeably, many scholars - across theoretical paradigms - find it useful, if not more accurate to maintain a difference between them (see González Alonso \& Rothman, 2017; Herdina \& Jessner, 2002; Paradis, 2004; Rothman, González Alonso and Puig-Mayenco, 2019; Schwartz \& Sprouse, 1996). Under such accounts, transfer sits at the level of mental representation, a copy from a previous language into the developing interlanguage grammar itself. Alternatively, CLIs are, as the name suggests, (in-the-moment) influences from other linguistic systems that bleed into performances of the non-native language for processing limitation or other specific reasons.

The above distinction is not merely a terminological one. Being at the level of mental representation, transfer relates directly to the learning task and developmental sequencing in non-native acquisition. That is, if a representation has been transferred from a previous linguistic system then this representation constitutes the point of departure for Ln acquisition. If the previous language has the same underlying representation, then transfer is facilitative, speeding up the overall acquisition of a given domain relative to child L1. If it is distinct, transfer is non-facilitative and can complicate, severely delay or render impossible the eventual acquisition (reconfiguration of the copied interlanguage representation in favor of the feature specification of the target) of the target grammar property. Whether facilitative or non- 
facilitative, transfer makes the point of departure specified, distinct from the state of underspecification that child L1 speakers enjoy. This is predicted to play out in developmental trajectories and potentially ultimate attainment differences from child L1, even if the adult sequential bilingual has access to the same underlying linguistic and cognitive mechanisms. Precisely because transfer sits at the level of grammatical representation, revealing and understanding how transfer plays out as well as its nature and timing are crucial for modeling the very learning task non-native speakers will undergo. Is it complete or partial? Is it wholesale (complete) at the very initial state/initial stages or does it obtain iteratively domain-by-domain throughout interlanguage development?

A related question of importance concerns the timing of and/or conditions under which transfer is best captured, that is, distinct from potential effects of target development/acquisition itself. After all, transfer is the initial interlanguage representation. Given exposure to the target language, development/acquisition takes place. And so, how can we be sure that what any given data reveal, seemingly showing evidence for or against specific theories of transfer, is in fact transfer proper and not already showing signs of a developing representation in the $\mathrm{L} n$ interlanguage? Conventional wisdom suggests that examining learners as close to the initial state of non-native acquisition as possible should help to reduce the noise in the signal of what we seek to capture. This truism has been robustly represented in the initial state hypotheses of L2 acquisition over time such as Full Transfer/Full Access (Schwartz \& Sprouse 1996); Minimal Trees Hypothesis (Vainikka \& Young-Scholten 1996); and the Valueless Features (Eubank 1994). Although many studies have used later-stage learners to comment on these initial state models, the most directly applicable evidence comes from studies that have groups with very limited exposure to the L2. This does not mean that transfer effects cannot linger into later stages of interlanguage development - in fact it can be predictively so if L1 transfer significantly complicates the L2 learning task-but failure to 
show evidence of transfer at a later stage in no way precludes it from having happened and already being overcome. The previous statement should be relatively uncontroversial in conceptual terms. To date, however, specific data underscoring this warning is not readily available. This brief article provides data that speak very directly to this possibility. Although they come from an instance of L3 acquisition, they embody a cautionary lesson with universal application for non-native language studies in a general sense. However, given the learner groups implicated we focus specifically on the direct implications for the emerging field of formal linguistic approaches to L3 acquisition, its theories and especially methodological practices.

\section{Adult Third Language (L3) Acquisition and Linguistic Transfer}

Over the past decade or so, there has been a sharp increase of research in adult third language (L3) acquisition from a multitude of perspectives. Questions related to L3 acquisition abound and can differ considerably across paradigms. Determining the relative role that previous linguistic experience plays in the unfolding of sequential multilingual acquisition, however, is a common theme across all approaches (e.g., Angelovska \& Hahn, 2017; Cenoz, Hufeisen, \& Jessner, 2001; De Angelis, 2007; De Angelis \& Dewaele, 2011; Rothman, Cabrelli Amaro, \& de Bot, 2013; Rothman \& Halloran, 2013). Within the nascent field of formal linguistic approaches to L3 acquisition, modeling the source (L1, L2 or both) of transferred representations into L3 (initial) interlanguage grammar(s) dominates. González Alonso \& Rothman (2017) and Rothman et al. (2019) discuss the manifold importance of such a question. They maintain that knowing what the initial stages grammar looks like is crucial for making informed/principled predictions for L3 developmental sequencing. Offering an analogy related to the strength of a building being proportional to the robustness and soundness of its foundation/basement, they argue that the success of meaningfully describing, predicting and 
explicating L3 development over time is also conditioned on the strength of understanding the target language's foundation, or the initial interlanguage. They also claim that transfer of mental representations - not mere influence from previous linguistic experience per se-is best studied at the L3 initial stages of true beginners, which is when transfer can be meaningfully teased apart from L3 learning/acquisition itself, because only at that stage can we be confident that representational transfer can be meaningfully teased apart from L3 learning/acquisition itself.

Existing models related to L3 morphosyntactic transfer have taken two main positions with respect to the above: multilingual transfer (a) defaults to the L1 or L2 or (b) does not default to either the L1 or L2; the selection depends on various factors hypothesized to motivate and/or delimit it. A-type models reflect the logical default possibilities-either the L1 (Hermas 2010; Hermas 2015) or the L2 (the L2 Status Factor: Bardel \& Falk, 2007, 2012; Falk, Lindqvist, \& Bardel, 2015)-has a privileged default status.

B-type models are necessarily more diverse; they must define how transfer unfolds (e.g., in whole or in parts) over time and what motivates transfer selectivity between the two available systems. B-type models can be divided into two main approaches, those that argue for holistic (full-system) transfer at the initial stages and those that argue for transfer obtaining property-by-property throughout development. The Typological Primacy Model (TPM: Rothman, 2010, 2011, 2015) stands out as the only B-type full transfer approach. The TPM maintains that the parser evaluates structural proximity between the target L3 input the learner receives at the initial stages against the $\mathrm{L} 1$ and $\mathrm{L} 2$. At the earliest possible moment, ${ }^{1}$ the parser

\footnotetext{
${ }^{1}$ Here, "earliest possible moment" refers to the point at which the parser has enough information from the L3 input stream to determine-via an implicit comparison to the L1 and L2 - which of the two complete systems is likely to be the best source of transfer. The "earliest possible moment" will vary along a continuum depending on the language triad in question, that is, based on the extent to which the L3 shares structural linguistic similarities at various levels of linguistic cues with both of the previously acquired languages and the extent to which one is likely to stand out over the other to the parser (see Rothman 2015; González Alonso \& Rothman, 2017 for detailed discussion).
} 
determines which whole system to transfer upon having had enough linguistic cue validity to make an informed selection (Rothman 2015; González Alonso \& Rothman 2017).

The other B-type models argue that transfer happens selectively throughout L3 development and is thus piecemeal; that is, it unfolds property-by-property over time conditioned by key factors that themselves differentiate these approaches. The Cumulative Enhancement Model (CEM: Berkes \& Flynn, 2012; Flynn, Foley, \& Vinnitskaya, 2004) argues that maximal facilitation motivates whether the L1 or L2 transfers; transfer obtains if and only if one provides a bootstrap for a given property in the L3. The Linguistic Proximity Model (LPM: Westergaard, Mitrofanova, Mykhaylyk, \& Rodina, 2017), alternatively, rejects the maximal facilitation notion but maintains that transfer happens domain-by-domain over the course of development and is motivated by the comparative linguistic proximity of the L3 to the other systems. Moreover, the LPM offers the possibility that both the L1 and L2 could have a simultaneously cumulative effect, giving rise to what looks like amalgamated or hybrid transfer. The Scalpel Model (SM: Slabakova, 2017) aligns with the LPM, however, adds a series of potentially ameliorating factors that might obscure the straightforward alignment of linguistic proximity.

In what remains, we provide some novel evidence to adjudicate between the above models, especially concerning whether transfer is complete (full transfer of an L1 or L2) or more likely reflects property-by-property transfer. No one denies the existence of ambiguous evidence across the literature that complicates answering this important question. However, we provide some data from an on-going project that shed important light on this debate, revealing that transfer can indeed appear piecemeal or to reflect hybridity when certain key variables are not controlled. In doing so, we underscore real value of this brief article, which serves as a methodological discussion for transfer research more generally and thus has further reach than L3 studies alone. 


\section{Study}

\subsection{Participants}

We consider data from both a superset $(n=60)$ and subset $(n=40)$ from that superset of L3 learners of English who are highly proficient Catalan-Spanish bilinguals of two types: L1 Catalan-L2 Spanish $(\mathrm{N}=35$, Mean Age $=54.4)$ and L1 Spanish-L2 Catalan $(\mathrm{N}=25$, Mean Age= 52.1). All participants came from a two-month language course $2 \mathrm{e}$ specifically designed and administered for beginners of L3English. The idea is that we would capture a large proportion of true ab initio L3 English learners, and, by designing our own course, we would be able to know and control the exact amount and type of L3 input they received. Even though all the enrolled had extremely low proficiency in English, there were participants with various degrees of previous exposure to English. We present two separate analyses: (i) the superset will all enrolled participants regardless of previous exposure to English and (ii) the subset of true ab initio learners without any previous exposure to English at all. In addition to the experiment introduced below, each participant completed the Oxford Quick Placement Test (all participants scored within the A1 level of the Common European Framework of Reference for Languages) and the Bilingualism Language Profile (BLP: Birdsong, Gertken, \& Amengual, 2012) - a questionnaire used to determine relative dominance and linguistic profile in bilinguals - to which we added several questions to ascertain any previous exposure to the L3, English, instruction or otherwise. Such questions were crucial for us to be able to isolate $a$ posteriori the ab initio beginners from low proficiency beginners who did have previous exposure.

\subsection{Linguistic Properties}


We tested various types of related constructions. This being a brief article and space thus being at a premium, we draw together results from two specific domains to make our points; the interpretation of Negative Quantifiers (NQs) and Negative Polarity Items (NPIs) in two different contexts: (a) in pre-verbal position with the presence of the sentential negative marker and (b) in (non-veridical) conditional contexts. The sentences examined here contained either negative quantifiers 'nobody/nothing' or negative polarity items 'anyone/ anything' in subject position of a transitive verb in context (a) as in (1)-(2) and in object position of transitive verbs for context (b) as in (3)-(4):

(1) \#Nobody does not drink coffee. (Acceptable only with Double Negation reading)

(2) *Anybody does not drink coffee.

(3) Laura will call us if Peter says nothing.

(4) Laura will call us if Peter says anything.

The choice of these constructions and these lexical items is interesting for two main reasons: (1) nobody/nothing and anyone/anything are interpreted differently in these contexts in English; and (2) Spanish and Catalan only have one lexical item to express both meanings, but crucially Catalan and Spanish give rise to different interpretations.

With respect to the English interpretations, sentence (1) can only give rise to a Double Negation Interpretation-effectively canceling semantic negation, or is otherwise ungrammatical. Sentence (2) is simply ungrammatical. In sentence (3), 'nothing' must have a negative reading and 'anything' in sentence (4) can only have an existential reading. Spanish and Catalan work differently. When the Negative Concord Item (NCI) occurs in in pre-verbal position with sentential negation, the Spanish version of the sentence also gives rise to a Double Negation reading as in (5), whereas the Catalan version gives rise to a single negation interpretation reading as in (6). 
(5) \#Nadie no bebe café.

Spanish

Nobody not drinks coffee

'Nobody does not drink coffee.' (DN reading)

(DN reading: 'There is nobody that does not drink coffee')

(6) Ningú no beu cafè

Nobody not drinks coffee

'Nobody drinks coffee.'

(SN reading: 'There is nobody that drinks coffee')

In a conditional context, Spanish nada 'nothing' (7) is interpreted with a negative reading as the English negative quantifier would be. Alternatively, Catalan res 'nothing' in this same context has the same interpretation as an English NPI, that is, it takes an existential reading $(8){ }^{2}$

(7) *Laura me llamará si Juan dice nada. Spanish Laura will.call.me if Juan says nothing

'Laura will call me if Juan says nothing.'

(Negative reading: 'Laura will call me if Juan says nothing')

(8) La Laura em trucarà si en Joan diu res. Catalan Laura will.call.me if Joan says nothing 'Laura will call me if Joan says nothing.'

(Existential reading: 'Laura will call me if Joan says anything at all')

\subsection{Task}

Herein we focus on the results of a Sentence-Picture Matching Interpretation task. The task presents target sentences and a choice between two pictures depicting the possible interpretations. In accord with the above descriptions, only one interpretation should be expected depending on the transfer source (Catalan or Spanish). The larger experiment comprised of 8 conditions with 4 experimental items, in addition to 32 distractor items. Given space limitations, it suffices that we focus on a subset of conditions ( 4 of 8 ) to reveal the

\footnotetext{
2 The reader is referred to the literature for specific analysis of NQs, NPIs in English and NCIs in Catalan and Spanish, (e.g., Espinal, 2000; Espinal \& Tubau, 2016; Haegeman \& Zanuttini, 1991; Tubau, 2008; Vallduví, 1994).
} 
empirical basis supporting the methodological point, as presented in Table 1 below. The other conditions we do not report here were the control conditions which work the same in Spanish and Catalan, ((i)Nobody...VERB, (ii)Anybody...VERB, (iii)VERB...nothing and (iv)VERB...anything), yet only 2 of 4 work the same in English (conditions (i) and (ii)). Obviously, these control conditions cannot reveal the transfer source, but given the grammatical versus ungrammatical asymmetry with English they serve as control conditions. All participants performed unsurprisingly, demonstrating a transfer effect.

Participants saw a sentence and two pictures depicting different interpretations. The conditions and example sentences we report on are summarized in the table below:

Table 1. Conditions, Example items and Interpretations.

\begin{tabular}{llcc}
\hline \multicolumn{1}{c}{ Condition } & Example & Picture A & Picture B \\
\hline Nobody...not $^{\mathrm{A}}$ & Nobody doesn't drink coffee. & $\begin{array}{c}\text { Double } \\
\text { Negation }\end{array}$ & $\begin{array}{c}\text { Single } \\
\text { Negation }\end{array}$ \\
\hline Anybody...not $^{\mathrm{A}}$ & Anybody doesn't drink coffee. & $\begin{array}{c}\text { Double } \\
\text { Negation }\end{array}$ & $\begin{array}{c}\text { Single } \\
\text { Negation }\end{array}$ \\
\hline Conditional...nothing $^{\mathrm{B}}$ & Mary will call us if Peter drinks nothing. & $\begin{array}{c}\text { Existential } \\
\text { Interpretation }\end{array}$ & $\begin{array}{c}\text { Negative } \\
\text { Interpretation }\end{array}$ \\
\hline Conditional....anything $^{\mathrm{B}}$ & Mary will call us if Peter drinks anything. & $\begin{array}{c}\text { Existential } \\
\text { Interpretation }\end{array}$ & $\begin{array}{c}\text { Negative } \\
\text { Interpretation }\end{array}$ \\
\hline
\end{tabular}

\footnotetext{
${ }^{A}$ For the if speakers interpreted the sentences with DN readings (Picture A), it would be evidence for Spanish transfer; if they interpreted these sentences with SN readings (Picture B), it would be evidence for Catalan transfer.

${ }^{B}$ For this context if speakers interpreted the sentences with negative readings (Picture B), it would be evidence for Spanish transfer; if they interpreted these sentences with existential readings (Picture A), it would be evidence for Catalan transfer.
}

Depending on the picture chosen for the condition - in accord with the Catalan or Spanish interpretations discussed above-we could determine which previous language was transferred. The following figure provides an example of one of the experimental items, indicating the expected language specific readings. 
Mary will call us if Peter says nothing.
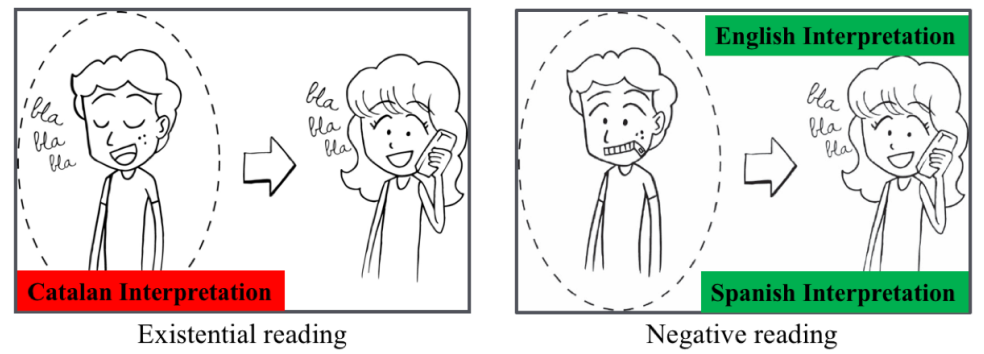

Figure 1 Example of the experimental item in the Conditional...nothing condition.

\subsection{Results: Superset analysis}

For the first two conditions (Nobody...not, Anybody...not), the results were coded as 1 for the Double Negation interpretation and 0 for the Single Negation interpretation readings. For the second two conditions (Conditional...nothing, Conditional... anything), the coding was 1 for the negative interpretation and 0 for the existential interpretation. See table 2 for raw counts and percentages.

Table 2. Raw counts and Percentages (\%) of the Double negation interpretations for the first two contexts and negative interpretations for the second two contexts (=Spanish-like interpretations)

\section{L1Catalan-L2Spanish L1Spanish-L2Catalan}

(35)

\begin{tabular}{lcccc}
\hline & Raw Counts & $\%$ & Raw Counts & $\%$ \\
\hline Nobody...NOT & $14 / 140$ & $10.0 \%$ & $22 / 100$ & $22.0 \%$ \\
\hline Anybody...NOT & $24 / 140$ & $17.1 \%$ & $14 / 100$ & $14.0 \%$ \\
\hline Conditional...nothing & $50 / 140$ & $35.8 \%$ & $38 / 100$ & $38.0 \%$ \\
\hline Conditional... anything & $14 / 140$ & $10.0 \%$ & $16 / 100$ & $16.0 \%$ \\
\hline
\end{tabular}

To understand the significance of the descriptive results in Table 2, we employed generalized linear mixed effects logistic regression analyses via the lme4 package (Bates, Maechler, Bokler, \& Walker, 2015) in the R environment (R Core Team, 2016). The two contexts (pre-verbal position with sentential negation vs. conditionals) were tested in distinct models due to the fact that they captured different interpretations: Nobody/Anybody...NOT conditions capture either Double Negation or Single Negation readings and the Conditional...nothing/anything conditions capture either negative readings or existential 
readings. The models tested the effects and interaction of Condition, Group and thus order of acquisition (L1Cat-L2Sp versus L1Sp-L2Cat) and Exposure to English (using months of exposure as a continuous variable, if applicable at the individual level) on the interpretations (coded as 1 and 0 ). The models include random by-participant by-item interecepts. The summaries of the omnibus models are presented in table 3.

Table 3. Generalized mixed effects models for the beginner learners.

\begin{tabular}{llll}
\hline Model Nobody/Anybody...NOT & & & \\
\hline $\begin{array}{l}\text { (Intercept) } \\
\text { (Ref: NQ+SN, L1Cat-L2SP) }\end{array}$ & Odds ratio & CI: LL, UL & $p$ \\
\hline L1-Spanish & 0.07 & $0.03,0.16$ & $<.001$ \\
\hline Condition:NPI+SN & 2.87 & $1.13,7.27$ & $<.001$ \\
\hline Exposure & 1.99 & $0.94,4.18$ & .069 \\
\hline L1SP*NPI+SN & 1.01 & $0.99,1.01$ & .531 \\
\hline
\end{tabular}

Model: Conditional...nothing/anything

\begin{tabular}{llll}
\hline $\begin{array}{l}\text { (Intercept) } \\
\text { (Ref: Con...any, L1Cat-L2SP) }\end{array}$ & 0.05 & $0.02,0.12$ & $<.001$ \\
\hline L1-Spanish & 1.95 & $0.57,6.60$ & .281 \\
\hline Condition:Con...nothing & 8.30 & $3.81,18.04$ & $<.001$ \\
\hline Exposure (months) & 1.02 & $1.01,1.05$ & $<.001$ \\
\hline L1SP*Con...nothing & 0.57 & $0.19,1.73$ & .329 \\
\hline
\end{tabular}

The first model shows that there is a significant main effect for group and a main interaction for group and condition. The L1Sp-L2Cat group gives more Double Negation readings to the Nobody...NOT condition than the L1Cat-L2Sp group $(p<.001)$ does. However, they do not differ for the Anybody...NOT condition $(p=.069)$. Even though the double negation readings are higher in this condition for the L1 Spanish group, they nevertheless also show transfer from Catalan because they only give Spanish-like interpretations (double negation readings) $17.1 \%$ of the time and Catalan-like ones (single negation readings) $82.9 \%$. 
When we examine the other model targeting the context with the conditional structures (Conditional...nothing, anything), it shows that there is a main effect for condition $(p<.001)$ and a main effect for exposure $(p<.001)$. It appears that both learner groups show Catalanlike interpretations (i.e., existential readings) for the negative polarity item when it is licensed by a conditional structure. Notice the low percentage of negative readings in this condition (L1-Cat: 10\%; L1-Sp: 16\%). Until now, having considered three of the four conditions under investigation, we have seen that all learners show Catalan transfer, irrespective of L1-L2 order of acquisition. However, when we explore the interpretation of negative quantifiers in conditional contexts, the picture is less clear. Irrespective of order of acquisition, both learner groups assign more negative interpretations to the negative quantifier than the negative polarity item (L1-Cat: 35.8\%; L1-Sp: 38\%). Thus, one might be tempted to conclude that for negative quantifiers, learners have a mixed initial representation reflecting hybrid transfer (i.e., an amalgamated influence) from both Catalan and Spanish.

Taken together, the data suggest that the models that propose a default status for the L1 or the L2 are on the wrong track, clearly indicating that transfer does not default to an L1 or L2. Furthermore, these data seemingly help to adjudicate between the models that do not suggest a default status, questioning the full transfer stipulation of the TPM in favor of models that predict the possibility of an amalgamated effect from both grammars, such as the LPM. Recall, however, that in the condition where there was apparent amalgamated influence of Catalan and Spanish, the effect the statistical model showed that exposure comes out as a significant predictor $(\mathrm{p}<.001)$ of individual variation. Despite the fact that all learners tested at very low English proficiency, the model reveals that the more exposure to L3 English one had prior to the specifically-designed course, the more negative readings were given to negative quantifiers in the conditional context. Clearly, if exposure comes out as a --in this case the only--significant predictor it can indicate, in accord with González Alonso and Rothman's 
warnings for L3 methodologies, probing for transfer beyond ab initio learners could have an inadvertent, obscuring effect. If exposure to the L3 beyond initial stages can indeed muddy the waters for determining initial stages transfer representations, even for relatively low L3 level proficiencies, the fact that some of the $60 \mathrm{~L} 3$ learners have had previous exposure to English might very well explain why the Conditional...nothing condition stands out as not showing clear transfer effects from one or the other language. Since ab initio trajectories reduce the possibility of confounding (L3) transfer effects with issues of (L3) interlanguage development itself, we ran the statistics again removing the low proficiency learners who were not ab initio prior to the course, which we present in the next section.

\subsection{Results: Subset analysis.}

Overall, 20 participants -13 from L1Cat-L2Sp and 7 L1Sp-L2Cat $-30.2 \%$ of the entire sample size were not $a b$ initio learners. These participants reported having taken part in short courses of English as a Foreign language (between 12 and 48 months) either in tutored or classroomsettings. Indeed, they were all beginners as shown in their proficiency but some had had slight to significant previous temporal exposure to English.

Excluding these 20 participants, the shape of the data is quite different as we see below. Table 4 already reveals that when true ab initio L3 learners are isolated two important changes happen: (a) the percentage of double negation interpretations for the L1Sp-L2Cat group for the Nobody...NOT condition drops from $22 \%$ to $9.7 \%$ and (b) the interpretation of negative quantifiers in the Conditional...nothing conditions drops from $35.8-38 \%$ to approximately $7.7 \%-12.8 \%$ for both groups, aligning these results with those of the negative polarity items.

Table 4. Raw counts and Percentages (\%) of the Double negation interpretations for the first two contexts and negative interpretations for the second two contexts (=Spanish-like interpretations) for the $a b$ initio learners only.

\begin{tabular}{crcr}
\hline \multicolumn{2}{c}{ L1Cat-L2Sp (23) } & \multicolumn{2}{c}{ L1Sp-L2Cat (18) } \\
\hline Raw Counts & $\%$ & Raw Counts & $\%$ \\
\hline
\end{tabular}




\begin{tabular}{lcccc}
\hline Nobody...NOT & $11 / 88$ & $12.5 \%$ & $7 / 72$ & $9.7 \%$ \\
\hline Anybody...NOT & $7 / 88$ & $7.95 \%$ & $12 / 72$ & $14.0 \%$ \\
\hline Conditional...nothing & $9 / 88$ & $10.22 \%$ & $10 / 72$ & $13.9 \%$ \\
\hline Conditional...anything & $7 / 88$ & $7.95 \%$ & $9 / 72$ & $12.8 \%$ \\
\hline
\end{tabular}

The same statistical models used above ${ }^{3}$ were applied on the dataset after excluding the 20 participants, as seen in table 5.

Table 5. Generalized mixed effects models for the ab initio learners.

\begin{tabular}{llll}
\hline Model Nobody/Anybody...NOT & & & \\
\hline & Odds ratio & CI: LL, UL & $p$ \\
\hline $\begin{array}{l}\text { (Intercept) } \\
\text { (Ref: NQ+SN, L1Cat-L2SP) }\end{array}$ & 0.12 & $0.05,0.27$ & $<.001$ \\
\hline L1-SP & 0.72 & $0.24,2.19$ & 0.572 \\
\hline Condition:Anybody...NOT & 0.53 & $0.19,1.45$ & 0.220 \\
\hline L1SP*Anybody...NOT & 2.85 & $0.66,12.16$ & 0.157 \\
\hline
\end{tabular}

Model: Conditional...nothing/anything

(Intercept)

\begin{tabular}{llll} 
(Ref: Con...any, L1Cat-L2SP) & 0.06 & $0.02,0.16$ & $<.001$ \\
\hline L1Sp & 2.02 & $0.62,6.57$ & 0.242 \\
\hline Condition:Con...nothing & 1.34 & $0.46,3.84$ & 0.593 \\
\hline L1SP*Con...nothing & 0.74 & $0.17,3.16$ & 0.694 \\
\hline
\end{tabular}

As a result, the evidence suggesting hybrid transfer disappears. Both groups now comprised of true $a b$ initio learners give existential readings to negative quantifiers and negative polarity items in a conditional sentence frame, which suggests that all ab initio learners interpret them as influenced from Catalan only, irrespective of whether Catalan is their L1 or L2.

\section{Discussion and Implications}

The above data provide empirical evidence warning that precipitous evaluations of models of initial stages morphosyntactic transfer in L3/Ln acquisition from data sets beyond ab initio L3

\footnotetext{
${ }^{3}$ Logically exposure is removed as a variable because as a matter of inclusion in these models exposure was kept constant across all individuals stemming from the 2-month course.
} 
learners need to proceed with caution. To our knowledge, this is the first dataset of its kind where, as a byproduct of the larger study design, we are able to retroactively look at the data in two ways to evaluate the tenability of the claim that data collected past the initial stages can make it difficult to distinguish what is transfer from what are effects of, in this case L3, developmental acquisition. As is true of the much wider studied case of adult L2 acquisition, showing what we have does not mean that looking at L3 acquisition data across various levels of increasing L3 exposure/proficiency, even for the purpose of cross-linguistic influence, is not something one wants to do. Quite the contrary. Like any other instance of acquisition, one wants to understand the L3 process from initial representations through development and ultimate attainment. The point here is simply one of what can be claimed based on particular data sets. As we saw, not considering the actual exposure of the participants but instead taking beginning proficiency as a proxy for exposure would have led to the conclusion that various models are rejected, that transfer is clearly not complete and more. Although these data do not prove that models that would be rejected without a more nuanced approach are in fact confirmed, they underscore the importance of using the right type of data to make certain claims, especially when rejecting a model in its entirety. And this methodological caveat, the main point of this brief article, is true ubiquitously for all studies of linguistic transfer be them in children or adults and irrespective of the quantity of languages involved. In the present case, the fact that beginning proficiency was shown to reflect some effects of L3 learning alreadywhen this coincided with non-ab initio learners-tells us that exposure to the L3 otherwise nullified by using proficiency as a catch-all proxy can inadvertently obscure answers to important, debated questions. If our participants with previous exposure to English present so differently from those who truly never had any exposure, one might question what this means for the vast majority of data in L3 studies that conflate beginning proficiency with true L3 initial stages. 


\section{References}

Angelis, Gessica De. 2007. Third or Additional Language Acquisition. Clevedon: Multilingual Matters.

Angelis, Gessica De \& Jean-Marc Dewaele. 2011. New Trends in Crosslinguistic Influence and Multilingualism Research. (Ed.) Gessica De Angelis \& Jean Marc Dewaele. Bristol: Multilingual Matters.

Angelovska, Tanja \& Angela Hahn. 2017. L3 Syntactic Transfer: Models, new developments and implications. (Ed.) Tanja Angelovska \& Angela Hahn. Amsterdam: John Benjamins.

Bardel, Camilla \& Ylva Falk. 2007. The role of the second language in third language acquisition: the case of Germanic syntax. Second Language Research 23(4). 459-484. doi:10.1177/0267658307080557.

Bardel, Camilla \& Ylva Falk. 2012. Behind the L2 Status Factor: A neurolinguistic framework for L3 research. In Jennifer Cabrelli Amaro, Suzanne Flynn \& Jason Rothman (eds.), Third Language Acquisition in Adulthood, 61-78. Amsterdam: John Benjamins.

Berkes, Éva \& Suzanne Flynn. 2012. Further evidence in support of the CumulativeEnhancement Model. In Jennifer Cabrelli Amaro, Suzanne Flynn \& Jason Rothman (eds.), Third Language Acquisition in Adulthood, 143-164. Amsterdam: John Benjamins.

Birdsong, David, Gertken, Libby M \& Mark Amengual. 2012. Bilingual Language Profile: An Easy-to-Use Instrument to Assess Bilingualism. COERLL. University of Texas.

Cenoz, Jasone, Britta Hufeisen \& Ulrike Jessner. 2001. Cross-Linguistic Influence in Third Language Acquisition: Psycholinguistics Perspectives. Clevedon, UK: Multilingual Matters.

Espinal, M Teresa. 2000. On the semantic status of n-words in Catalan and Spanish. Lingua 110. 557-580.

Espinal, M Teresa \& Susagna Tubau. 2016. Meaning of words, meaning of sentences. Building the meaning of n-words. In S Fischer \& C Gabriel (eds.), Manual of grammatical interfaces in Romance, 187-212. Berlin, Germany: De Gruyter.

Eubank, L. 1994. On the transfer of parametric values in L2 development. Language Acquisition 3. 183-208.

Falk, Ylva, Christina Lindqvist \& Camilla Bardel. 2015. The role of L1 explicit metalinguistic knowledge in L3 oral production at the initial state. Bilingualism: Language and Cognition 18(2). 227-235. doi:10.1017/S1366728913000552. http://www.journals.cambridge.org/abstract_S1366728913000552.

Flynn, Suzanne, Claire Foley \& Inna Vinnitskaya. 2004. The Cumulative-Enhancement model for language acquisition: Comparing adults' and children's patterns of development in first, second and third language acquisition. International Journal of Multilingualism 1(1). 3-16. http://www.tandfonline.com/doi/abs/10.1080/14790710408668175 (29 July, 2014).

González Alonso, Jorge \& Jason Rothman. 2017. Coming of age in L3 initial stages transfer models: Deriving developmental predictions and looking towards the future. International Journal of Bilingualism 21(6). 683-697. doi:10.1177/1367006916649265. http://ijb.sagepub.com/cgi/doi/10.1177/1367006916649265.

Haegeman, Liliane \& Raffaella Zanuttini. 1991. Negative heads and the Neg criterion. Linguistic Review 8(2). 233-252.

Herdina, Philip \& Ulrike Jessner. 2002. A Dynamic Model of Multilingualism: Perspectives of Change in Psycholinguistics. Bristol, UK: Multilingual Matters.

Hermas, Abdelkader. 2010. Language acquisition as computational resetting: verb movement in L3 initial state. International Journal of Multilingualism 7(4). 343-362. 
doi:10.1080/14790718.2010.487941.

http://www.tandfonline.com/doi/abs/10.1080/14790718.2010.487941.

Hermas, Abdelkader. 2015. The categorization of the relative complementizer phrase in thirdlanguage English: A feature re-assembly account. International Journal of Bilingualism 19(5). 587-607.

Paradis, Michel. 2004. A Neurolinguistic Theory of Bilingualism. Amsterdam: John Benjamins.

Rothman, Jason. 2010. On the typological economy of syntactic transfer: Word order and relative clause high/low attachment preference in L3 Brazilian Portuguese. International Review of Applied Linguistics in Language Teaching (IRAL) 48(2-3). 245-273. doi:10.1515/iral.2010.011.

Rothman, Jason. 2011. L3 syntactic transfer selectivity and typological determinacy: The Typological Primacy Model. Second Language Research 27. 107-127. doi:10.1177/0267658310386439.

Rothman, Jason. 2015. Linguistic and cognitive motivations for the Typological Primacy Model (TPM) of third language (L3) transfer: Timing of acquisition and proficiency considered. Bilingualism: Language and Cognition 18(2). 179-190. doi:10.1017/S136672891300059.

Rothman, Jason, Jennifer Cabrelli Amaro \& Kees de Bot. 2013. Third Language Acquisition. In Julia Herschensohn \& Martha Young-Scholten (eds.), The Cambridge Handbook of Second Language Acquisition, 372-393. Cambridge, UK: Cambridge University Press.

Rothman, Jason, González Alonso, Jorge \& Eloi Puig-Mayenco. 2019. Third Language Acquisition and Linguistic Transfer. Cambrige: Cambridge University Press.

Rothman, Jason \& Becky Halloran. 2013. Formal Linguistic Approaches to L3/Ln Acquisition: A Focus on Morphosyntactic Transfer in Adult Multilingualism. Annual Review of Applied Linguistics 33. 51-67. doi:10.1017/S0267190513000032.

Schwartz, Bonnie \& Rex Sprouse. 1996. L2 cognitive states and the Full Transfer/Full Access model. Second Language Research 12(1). 40-72. doi:10.1177/026765839601200103.

Slabakova, Roumyana. 2017. The Scalpel Model of Third Language Acquisition. International Journal of Bilingualism. Tromsø, Norway 21(6). 651-666. doi:10.1177/1367006916655413.

http://ijb.sagepub.com/cgi/doi/10.1177/1367006916655413.

Tubau, Susagna. 2008. Negative Concord in English and Romance: Syntax-morphology interface conditions on the expression of negation. Utrecht, NL: LOT Publications.

Vainikka, Anne \& Martha Young-Scholten. 1996. The early stages in adult L2 syntax: additional evidence from Romance speakers. Second Language Research 12(2). 140-176.

Vallduví, Enric. 1994. Polarity items, n-words and minimizers in Catalan and Spanish. Probus 6. 263-294.

Westergaard, Marit, Natalia Mitrofanova, Roksolana Mykhaylyk \& Yulia Rodina. 2017. Crosslinguistic influence in the acquisition of a third language: The Linguistic Proximity Model. International Journal of Bilingualism 21(6). 666-682. doi:DOI: $10.1177 / 1367006916648859$.

White, Lydia. 1989. Universal Grammar and Second Language Acquisition. Amsterdam: John Benjamins Publishing Company.

White, Lydia. 2003. Second Language Acquisition and Universal Grammar. Cambridge textbooks in linguistics. Cambridge, UK: Cambridge University Press. 